\title{
A marine fungus efficiently degrades polyethylene
}

\author{
Rongrong Gao ${ }^{\mathrm{a}, \mathrm{b}, \mathrm{c}, \mathrm{d}}$, Rui Liu ${ }^{\mathrm{a}, \mathrm{b}, \mathrm{d}}$, Chaomin $\operatorname{Sun}^{\mathrm{a}, \mathrm{b}, \mathrm{c}, \mathrm{d}^{*}}$
}

$3 \quad{ }^{\mathrm{a}} \mathrm{CAS}$ and Shandong Province Key Laboratory of Experimental Marine Biology \&

4 Center of Deep Sea Research, Institute of Oceanology, Chinese Academy of

5 Sciences, Qingdao, China

$6 \quad{ }^{\mathrm{b}}$ Laboratory for Marine Biology and Biotechnology, Pilot National Laboratory for

7 Marine Science and Technology, Qingdao, China

$8{ }^{\mathrm{c}}$ College of Earth Science, University of Chinese Academy of Sciences, Beijing,

9 China

$10{ }^{\mathrm{d}}$ Center of Ocean Mega-Science, Chinese Academy of Sciences, Qingdao, China

11

$12 *$ Corresponding author

13

14

$$
\text { Chaomin Sun Tel.: +86 } 532 \text { 82898857; fax: +86 } 53282898857 \text {. }
$$

15

16

17 Keywords: Ocean, fungus, polyethylene, biodegradation, enzymes

18 Running title: A marine fungus degrades polyethylene

19

20

21

22

23

24

25

26 


\section{Abstract}

28 Plastics pollution has been a global concern. Huge quantities of polyethylene (PE), the 29 most abundant and refractory plastic in the world, have been accumulating in the 30 environment causing serious ecological problems. However, the paucity of 31 microorganisms and enzymes that efficiently degrading PE seriously impedes the 32 development of bio-products to eliminate this environmental pollution. Here, by 33 screening hundreds of plastic waste-associated samples, we isolated a fungus (named 34 Alternaria sp. FB1) that possessing a prominent capability of colonizing, degrading 35 and utilizing PE. Strikingly, the molecular weight of PE film decreased $95 \%$ after the 36 fungal treatment. Using GC-MS, we further clarified that a four-carbon product 37 (named Diglycolamine) accounted for $93.28 \%$ of all degradation products after the 38 treatment by strain FB1. We defined potential enzymes that involved in the 39 degradation of PE through a transcriptomic method. The degradation capabilities of 40 two representative enzymes including a laccase and a peroxidase were verified. Lastly, 41 a complete biodegradation process of PE is proposed. Our study provides a 42 compelling candidate for further investigation of degradation mechanisms and 43 development of biodegradation products of PE. 


\section{Introduction}

56 Plastic deposition has accumulated tremendously due to its extensive production, widespread application and high resistance to biodegradation [1-3]. The global

58 production has expanded to 464 million tons in 2018 [4], and 50\% of them was

59 discarded within a short period after use[4, 5]. Among the total accumulated plastic waste, polythene (PE) alone accounts for $64 \%[6,7]$, and is considered as most ecological problematic due to its high molecular weight, strong hydrophobicity and highly inert chemically and biologically [8-12]. In this sense, it is an urgent need to find an efficient approach to degrade the PE waste for decontamination from the environment. The degradation of PE can occur by chemical, thermal, photo or biological degradation [13]. Recently, biodegradation using microorganisms has become a promising alternative for plastic recycling due to the mild and environmentally friendly reaction conditions required [14].

Among these microbes, fungi are potentially effective for the degradation of environmental PE [15-18]. Generally, fungi as well as other microbes are involved in

70 the depolymerization, assimilation and mineralization processes of plastic degradation 71 along with the involvement of a set of enzymatic system responsible for diverse steps 72 including colonization, degradation, transformation and utilization [19-21]. The 73 hydrophilic surfaces make the initial colonization on PE surface really difficult, 74 however, microbial enzymes can efficiently promote the attachment of 75 microorganisms to the surface of PE by improving the plastic hydrophilicity [22]. 76 Once fungi colonize on the surface of PE, the degradation and utilization processes 77 could be performed in combination with both intracellular and extracellular enzymatic 78 systems, which enable them to use complex polymers as a source of carbon and 79 electrons for further growth [16]. The extracellular enzymatic system consists of the 80 hydrolytic system that formed mainly by nonspecific oxidoreductases, including 81 versatile peroxidases, laccases, and unspecific peroxygenases [23]. The intracellular 
82 enzymatic system is normally mediated by the cytochrome P450 family epoxidases 83 and transferases [24].

84 Thus far several fungi including Aspergillus, Acremonium, Fusarium, Penicillium,

85 Phanerochaete [25], and corresponding enzymes including laccases, manganese

86 peroxidase and lignin peroxidases [26], have been reported to potentially degrade PE.

87 Although some progresses have been made about fungi-mediated PE degradation, the

88 biocatalytic degradation of PE needs to be detailedly elucidated, especially the

89 category and function of enzymes responsible for the whole degradation process.

90 Further research is required on novel isolates from plastisphere ecosystems to deeply

91 disclose the associated mechanisms about PE biodegradation process [16].

92 Here, using a large-scale screening approach, we isolated and defined a marine

93 fungus, Alternaria sp. FB1, which could effectively colonize and degrade PE. Using

94 various techniques, we further clarified the degradation effects and released products.

95 Lastly, we discovered 153 potential enzymes associated with PE degradation via a

96 transcriptomic approach, and verified the degradation effects of two representative

97 enzymes. Lastly, we described the whole process of PE degradation and utilization

98 mediated by strain FB1.

\section{Results and Discussion}

\section{Discovery of a marine fungus that efficiently colonizes and degrades PE}

101 To obtain microorganisms possessing capability of degrading PE from marine

102 environments, about 500 sedimentary samples containing plastic contaminants were

103 collected from different locations of Huiquan bay of Qingdao, China. Thereafter,

104 these samples were respectively put into a flask filled with sterilized sea water

105 supplemented with only commercial PE bag fragments as the sole nutrient source.

106 After one-month incubation at $25^{\circ} \mathrm{C}$, in the flask incubated with the $6^{\text {th }}$ sample,

107 obvious filament bundles attached to four corners of the PE film were observed,

108 suggesting some fungi might be enriched from this sample. These filament bundles 
were thus removed from the PE film and observed through a light microscopy. Indeed,

110 the filament bundles attached to the PE film showed typical characteristics of fungal

111 hyphae. Thereafter, the filament bundles were cultured in the PDA medium for further

112 purification. After several rounds of purification and ITS (Internal Transcribed Spacer)

113 gene sequencing confirmation, one pure fungal strain named FB1 was isolated. The

114 fungal strain FB1 showed high homology (higher than 99\% identity) of ITS sequence

115 with many Alternaria strains, which was further confirmed by phylogenetic tree

116 analysis (Fig. S1). Considering no more physiological characteristics were

117 investigated, the strain FB1 was designated as Alternaria sp. FB1 in this study.

118 Consistent well with its capability of growing in the sea water supplemented with only

119 PE bags, it is indeed capable of efficiently colonizing on the pure PE films after 3-day

120 incubation with PE in the seawater (Fig. 1b). Moreover, the fungus could generate

121 many substances similar to biofilm among different hyphae (Fig. 1c) and produce

122 large amount of spores for further multiplication (Fig. 1d), strongly suggesting strain

123 FB1 could utilize PE as a nutrient source for growth given the presence of very little

124 organic matter in the sea water. Consistently, strain FB1 showed a much better growth

125 status and stronger reproduction ability in the seawater supplemented with PE film

126 than that in the seawater only (Figs. S2-S3), confirming its capability of using PE as a

127 nutrient source for growth.

128 Next, after removing the microbial layer, we observed numerous holes in the PE 129 surface (Fig. 1f), strongly indicating strain FB1 could effectively degrade PE films.

130 The diameter of some holes could reach $0.5 \mu \mathrm{m}$, and some holes almost penetrated 131 across the film (Figs. 1g-h). When we extended the treatment time to four months, the 132 PE film could be thoroughly occupied by fungal hyphae and spores (Fig. 2c). The 133 color of PE film changed from white to yellow and black, and the morphology of PE 134 film became extreme curling and shrinking (Fig. 2c). On the other hand, while we 135 only dropped some fungal cells at a special spot of the PE film and incubated in the 136 sea water for about four months, almost all the growing area of fungus could separate 
137 from the original PE film (Figs. 2d-e). Taken together, we are confident that the

138 marine fungus Alternaria sp. FB1 possesses prominent capabilities of colonizing,

139 degrading PE and thereby utilizing as a nutrient source for growth.

140 Recently, it has been calculated that a range of 4.8-12.7 million tons of plastics

141 enter the oceans annually [27], thus plastic pollutions have been recognized as the

142 most common and durable marine contaminants. Consequently, the marine

143 environment is becoming a hot spot to screen microorganisms possessing prominent

144 plastic degradation capabilities [28], and our recent [29] and present works confirm

145 the proposal. It is noting that Alternaria sp. FB1 is a typical representative of

146 filamentous fungi that are found in different environments and some of them have

147 evolved to adapt and grow even in terrestrial and marine environments under extreme

148 conditions [16]. In particular, fungi are able to extend through substrates in their

149 search for nutriments with their filamentous network structure, exploring and growing

150 in places that are more difficult to reach for other microorganisms [16]. Indeed, our

151 results clearly show that strain FB1 not only penetrates the PE film (Fig. 2) but also

152 extends its growing location all over the surface of plastic (Fig. 1c), indicating fungi

153 are good candidates for developing PE degradation bio-products.

154 Verification of PE degradation effects conducted by Alternaria sp. FB1

155 Generally, several approaches are adopted to roughly evaluate visible changes in PE

156 degradation, such as the formation of surface biofilms, holes, cracks, fragmentation,

157 color changes, and surface roughness. The level of PE degradation can be further

158 determined by SEM to verify the level of scission and attachment of the

159 microorganisms, by Fourier Transform Infrared (FTIR) to analyze the

160 microdestruction of the small samples, by X-Ray Diffraction (XRD) to evaluate the

161 crystallinity degree, by Gel Permeation Chromatography (GPC) to estimate the 162 depolymerization of PE long-chain structure [29, 30]. Next, we sought to further

163 verify the PE degradation effects conducted by Alternaria sp. FB1 through above

164 various approaches. First, FTIR analysis was conducted to detect the degradation 
165 effects. Compared to the control group, two extra FTIR spectra absorption peaks were 166 observed in a 2-week fungus treated PE film (Fig. 3a, green curve). One absorption 167 peak was observed in the vicinity of $1,715 \mathrm{~cm}^{-1}$, indicating the formation of carbonyl 168 bonds $(-\mathrm{C}=\mathrm{O}-)$, while the other absorption peak was observed at a wave number of $1693,318 \mathrm{~cm}^{-1}$ and was attributed to hydroxyl groups. Moreover, the signal strength of 170 above two peaks became much stronger when the treatment time was extended to four 171 weeks (Fig. 3a, red curve). According to these key chemical bonds, we conclude that 172 PE film treated by Alternaria sp. FB1 underwent major structural changes 173 representing direct biodegradation by the fungus. Fungal treatment resulted in a 174 cleavage of the PE polymer chain, which thereby reducing the molecular weight and 175 increasing hydrophilicity of PE polymer.

176 On the other hand, through XRD analysis, we found that PE film treated by 177 strain FB1 for 28 day s showed an evident reduced relative crystallinity degree, as 178 measured by peak-differentiating and imitating calculations, resulting in a decrease 179 from $62.79 \%$ to $52.02 \%$ (Fig. 3b). The XRD results clearly indicate that fungal 180 treatment could significantly change the structure of molecular arrangement of PE 181 polymer. Lastly, GPC was performed to determine the number-average molecular 182 weight $(\mathrm{Mn})$, molecular weight (Mw) and molecular weight distribution (MWD) of 183 fungus treated PE films, which are three key indicators of the scission and degradation 184 of plastics. After a 120-day treatment, the Mns of fungus-treated PE and medium185 treated PE were respective 3,223 and 29,218, leading to a 9-fold decrease; the Mws of 186 fungus-treated PE and culture-treated PE were respective 1,1959 and 231,017, 187 resulting in a 20-fold decrease. Consistently, the MWD of fungus-treated PE (Fig. 3d) 188 showed a markedly decrease trend compared to the culture-treated PE (Fig. 3c). The 189 decrease of MWD and increase of the proportions of lower molecular weight 190 fragments strongly suggested the occurence of depolymerization of the PE long-chain 191 structure. 
193 Penicillium as well as Fusarium were found to be potentially efficient for PE

194 degradation based on weight loss, molecular weight decrease and reduction in tensile

195 strength [18]. In contrast, only one fungus belonging to the genus Alternaria was

196 reported to cooperate with other fungi within a consortium to degrade the PE film [31].

197 Actually, the genus Alternaria includes more than 250 species and is ubiquitously

198 distributed in diverse terrestrial and marine environments [32]. Our study clearly

199 shows that Alternaria sp. FB1 possesses a prominent capability of degrading PE film:

200 the molecular weight of PE film could be decreased 95\% after fungal treatment,

201 indicating this fungal strain as well as other Alternaria members has great potentials

202 to develop plastic degradation products.

203 Analysis of PE degradation products by Gas Chromatography-Mass 204 Spectrometer (GC-MS) analysis

205 To further explore the details of PE degradation conducted by strain Alternaria sp.

206 FB1, the degradation products were analyzed by GC-MS. For the 60-day treatment

207 sample, the major retention time peaks corresponding to $17.58 \mathrm{~min}, 16.70 \mathrm{~min}, 18.98$

$208 \mathrm{~min}, 15.69 \mathrm{~min}$ and $17.16 \mathrm{~min}$ are the top 5 based on area percent calculation (Fig.

209 S4). In contrast, for the 120-day treatment sample, only one predominant retention

210 time peak corresponding to 7.75 min was shown, accounting about $93.28 \%$ of all

211 peaks' area (Fig. S5). Next, constituents existing in the above six retention time peaks

212 were further identified by MS. The results revealed that within the 60-day treated

213 sample the carbon numbers of each product ranged from 12 to 30 (Fig. 4a,

214 Supplementary Table S1), and the product (1-monolinoleoylglycerol trimethylsilyl

215 ether) possessing 27 carbons was predominant, accounting for $51.24 \%$ of all products

216 (Fig. 4c). The rest predominant products were hexanedioic acid bis(2-ethylhexyl)

217 ester (16.42\%), squalene (13.89\%), tributyl phosphate (7.1\%), cycloheptasiloxane

218 tetradecamethyl- (3.45\%), cyclohexanamine N-cyclohexyl- (2.33\%), 13-Docosenoic

219 acid methyl ester, (Z)- (7.9\%) (Fig. 4a and Supplementary Table S1). In contrast, in 
220 the 120-day treated sample, the carbon number of corresponding products ranged

221 from 3 to 27 (Fig. 4b and Supplementary Table S2), and the product (Diglycolamine)

222 possessing 4 carbons was the most predominant one, accounting for $93.28 \%$ of all

223 products (Fig. 4c). Obviously, the proportion of product possessing smaller molecular

224 weight significantly increased along with the extension of treatment time from 60 to

225120 days, strongly suggesting that more evident degradation occurred after 120-day

226 treatment by strain FB1.

227 Although some fungal strains have been identified as candidates for PE 228 degradation[18], the degradation products are yet obscure. Notably, after $120 \mathrm{~d}$ 229 treatment by strain FB1, the predominant degradation product is identified as 230 Diglycolamine that possessing only four carbons (Fig. 4c). Diglycolamine, one of the 231 alkanolamine solvents, produces total organic acid anions as degraded products[33, 232 34], which might contribute to the energy metabolism by some unknown pathway.

233 We are confident that Diglycolamine is not a fungal metabolic product based on 234 different database searches, however, it is still not clear how does Diglycolamine 235 derive from the PE long chain and whether it will be degraded further or directly 236 utilized by the fungus. Nevertheless, our study provides a hint for researchers to 237 explore the degradation products of PE in the future.

\section{Transcriptomic profiling of the plastic degradation process}

239 These parameters obtained from the FTIR, XRD, GPC as well as GC-MS can be used 240 as indicators of microbial action, however, these results do not reflect the metabolic 241 responses of microorganisms. To explore the plastic degradation process and potential 242 mechanisms mediated by strain FB1, we performed a transcriptome analysis of this 243 fungus cultured in the medium supplemented either with or without PE for 45 days. 244 Combined with our gene expression analyses, we discovered 153 potential enzymes 245 closely associated with biodegradation and the expressions of their encoding genes 246 were significantly upregulated (Fig. 5a). In summary, these enzymes include 3 247 peroxidases, 3 laccases, 26 hydroxylases (4 hydroxylases, 15 monooxygenases, 7 
248

249

250

251

252

253

254

255

256

257

258

259

260

261

262

263

264

265

266

267

268

269

270

271

272

273

274

275

oxygenases), 49 dehydrogenases, 18 oxidoreductases, 10 oxidases, 22 reductases, 16 esterases, 4 lipases and 2 cutinases. In particular, the transcription levels of laccase encoding gene (Gene id: evm.TU.contig_8.535), peroxidase encoding gene (Gene id: evm.TU.contig_5.872) and oxidoreductase encoding gene (Gene id: evm.TU.contig_5.292) were respectively increased about 23, 44 and 102 folds when compared the expressions of these genes under conditions supplemented with or without PE, strongly suggesting the key role s of these enzymes in the process of PEdegradation mediated by strain FB1.

To further verify the transcriptomic results, we overexpressed two putative PE degrading enzymes including glutathione peroxidase (evm.model.contig_3.359) and laccase (evm.model.contig_8.535) in E. coli cells (Fig. S26), and checked their respective degradation effects on PE films in $48 \mathrm{~h}$. Notably, these two enzymes showed degradation effects on PE films compared to the control (treated by sterile seawater, Fig. 5b), obvious cracks and signs of plastic film degradation were observed by the SEM (Figs. 5c-d). Especially, glutathione peroxidase and laccase showed a clear synergetic degradation effect on the PE film (Fig. 5e). Consistently, the GPC analyses toward to both $\mathrm{Mn}$ and $\mathrm{Mw}$ of PE films treated by above two enzymes alone or together showed similar patterns to those of SEM observations. That is, the combined utilization of two enzymes led to a much higher degradation rate than those of single enzyme (Figs. 5f-i). The Mn and $\mathrm{Mw}$ of the PE film treated by both glutathione peroxidase and laccase were respective 20904 and 109202, which showed about $18 \%$ and $7 \%$ (Fig. 5i) decreases compared to those of control (Mn and $\mathrm{Mw}$ are 25516 and 116240, respectively, Fig. 5f). Future studies are required to test the degradation effects of more enzymes revealed by the transcriptomic results and develop a combined enzyme system for highly effective degradation of PE. Taken together, these knowledges greatly facilitate the protein and strain engineering for enhanced PE degradation performance, to meet the requirements for future industrial applications. 


\section{A proposed model of biodegradation process of PE}

277 Based on the combination of our genomic and transcriptomic data as well as previous

278 reports, we propose a detail PE-degradation process (Fig. 6). Briefly, the process of

279 PE biodegradation can be divided into four stages: colonization/corrosion,

280 depolymerization, assimilation and mineralization [25, 35]. In the colonization stage,

281 individual species or microbial consortium form a biofilm attached on the PE surface

282 [25]. Due to the interaction with the various extracellular enzyme produced by

283 microorganisms, the polymer surface was deteriorated and its hydrophobicity

284 undermined. Then the long chain of the polymeric structure was broken down and

285 was cut into small fragment by the action of a series enzymes secreted by the fungus.

286 The initial and rate-determining step is the oxidation of PE by some oxidative

287 enzymes such as peroxidase, oxygenase and laccase [15, 36, 37], which leading to a

288 reduction of molecular weight. After the oxidation, the PE polymer is destructed, the

289 molecular weight decreases, and carbonyl groups are introduced along the

290 polyethylene chain. The decrease of molecular weight enables transport of PE small

291 chain molecules through the cell membrane, it also makes degradation intermediates

292 easier to be recognized and attacked by fungal enzymatic systems such as hydroxylase,

293 monooxygenase, oxygenase, dehydrogenases, oxidoreductases esterases, lipases as

294 well as cutinases. Given the chemical similarity between PE and alkanes, it has been

295 suggested that the metabolic pathways for degradation of alkanes and PE are highly

296 similar once the size of PE molecules decrease to an acceptable range for enzyme

297 attack [25, 38]. In this sense, the PE degradation intermediates are further catalyzed

298 by terminal oxidation monooxygenase to alcohol, which is further oxidized by alcohol

299 and aldehyde dehydrogenases [39], and the resulting fatty acids enter the $\beta$-oxidation

300 cycle. In parallel, the PE degradation intermediates are also catalyzed by sub-terminal

301 oxidation monooxygenase to secondary alcohols, which are oxidized to ketones by

302 alcohol dehydrogenase. A Baeyer-Villiger monooxygenase converts ketones to esters,

303 which are subsequently cleaved by an esterase, cutinase and lipase. This leads to the 
304 formation of fatty acids and then degraded by $\beta$-oxidation. In the assimilation process,

305 some small water-soluble intermediates with short chains produced by

306 depolymerization are recognized by the receptors and then transported across the

307 membrane into the microorganism, and thereby participating in a variety of metabolic

308 activities and contributing to the cell growth. Finally, some metabolites and the non-

309 assimilated products generated in the assimilation process are completely absorpted

310 and utilized in mineralization, and are further converted to energy, carbon source as

311 well as the $\mathrm{CO}_{2}$ and $\mathrm{H}_{2} \mathrm{O}$.

\section{Conclusions}

313 In our present study, we successfully obtained a marine fungus, Alternaria sp. FB1,

314 which can efficiently colonize and degrade PE through forming numerous holes that 315 across the film. Through SEM, FTIR and XRD approaches, we systematically verified 316 the typical degradation indications including colonization, scission as well as 317 microdestruction of PE film treated by strain FB1. Using GPC assay, we estimated the 318 depolymerization of PE long-chain structure and find the molecular weight of PE film 319 was decreased $95 \%$ after fungal treatment. Using GC-MS, we further clarified that a 320 four-carbon product Diglycolamine was the most predominant (accounting for $93.28 \%$ 321 of all products) degradation product after 120 days treatment by strain FB1. We 322 defined the responses of this fungus directing plastic degradation through a 323 transcriptomic method, showing the expressions of genes encoding 153 potential 324 enzymes (including 3 peroxidases, 3 laccases, 26 hydroxylases, 49 dehydrogenases, 32518 oxidoreductases, 10 oxidases, 22 reductases, 16 esterases, 4 lipases and 2 cutinases) 326 are significantly up-regulated. The degradation effects of two representative enzymes 327 revealed by the transcriptomic method were further verified by both SEM and GPC 328 approaches. Lastly, three potential steps (including colonization, depolymerization 329 and assimilation/mineralization) involved in biodegradation of PE are proposed. 
331 PE plastics used for different assays. Three kinds of PE plastic are used in this study, 332 including commercial PE bags, type ET311350 PE plastic (0.25 mm in thickness) and 333 type ET311126 PE plastic (0.025 mm in thickness), the latter two are additive-free 334 plastic films and are purchased from the Good Fellow Company (UK). All PE films 335 are treated with $75 \%$ ethanol and air-dried in a laminar-flow clean bench prior to use.

336 Screening, isolation and identification of marine microorganisms capable of 337 degrading PE. To screen marine microorganisms capable of degrading PE, roughly 338500 plastic debris samples were collected from the intertidal locations in the Huiquan 339 Bay (Qingdao, China), and kept in flasks supplemented with filtered sea water and 340 commercial PE films at room temperature (about $25^{\circ} \mathrm{C}$ ) for different periods. During 341 this course, the films were checked by eyes and those covered by bacterial biofilm or 342 fungal hyphae were observed by light microscopy to confirm the colonization of 343 microorganisms. PDA medium (potato $200 \mathrm{~g}$, glucose $20 \mathrm{~g}$, agar 15 20 g, distilled 344 water $1000 \mathrm{~mL}$, natural $\mathrm{pH}$ ) was used to purify the fugal strain FB1, and its purity was 345 confirmed by PCR with the primers (ITSF: 5'-TCCGTAGGTGAACCTGCGG-3'; 346 ITSR: 5'-TCCTCCGCTTATTGATATGC-3') for identifying fungal ITS sequence. 347 After the fungus was purified, the minimal medium $(0.005 \mathrm{~g}$ yeast extract, $0.01 \mathrm{~g}$ 348 peptone, $0.002 \mathrm{~g}$ xylose in $1 \mathrm{~L}$ filtered and sterile seawater, $\mathrm{pH}$ 7.0) was utilized for 349 all growth and degradation assays of strain FB1 if not specified.

350 Microscopic observation. The morphology and colonization of fungus on the PE 351 film were observed and photographed by an inverted microscope (NIKON TS100, 352 Tokyo, Japan) or scanning electron microscope (Hitachi S-3400N, Japan). The fungus 353 or PE films were routinely observed by the inverted microscope according to the 354 instruction. To observe the colonization of fungus on the plastic, PE films treated by 355 medium or strain FB1 were soaked in 5\% glutaraldehyde for cell fixation and were 356 then dehydrated with 30\%, 50\%, 70\%, 90\%, 100\% graded ethanol for 10 min each 357 and critical-point-dried with $\mathrm{CO}_{2}$. Dried specimens were sputter coated for 5 min with 358 gold and platinum (10 nm) using a Hitachi MC1000 Ion Sputter (Japan). To observe 
359 the plastic degradation effects, medium- or fungus treated PE films were washed in

360 ultrasonic cleaner with $3 \% \mathrm{H}_{2} \mathrm{O}_{2}, 75 \%$ ethanol, and then distilled water to remove the

361 biofilms thoroughly [40]. And the observation by SEM was performed as described

362 above.

363 Fourier Transform Infrared (FTIR) analysis. For FTIR analysis, PE films were

364 recovered after a 2 -week or 4 -week fungus- or medium treatment. Films were then

365 successively rinsed in ultrasonic cleaner with $1 \%$ SDS, distilled water, and then $75 \%$

366 ethanol [41, 42]. After air drying, PE films were recorded over the wavelength range

367 of $450-4000 \mathrm{~cm}^{-1}$ at a resolution of $1 \mathrm{~cm}^{-1}$ using a Nicolet-360 FTIR (Waltham, USA)

368 spectrometer operating in ATR mode [43]. Thirty two scans were taken for each

369 spectrum.

370 X-Ray Diffraction (XRD) analysis. XRD was performed using a Bruker D8

371 Advance instrument with a wavelength of 1.5406 angstrom of $\mathrm{CuK} \alpha$ ray. The XRD

372 tube current was set as $40 \mathrm{~mA}$, and the tube voltage was set as $40 \mathrm{kV}$. Measurements

373 for PE were set in the angle range from $2 \theta=3^{\circ}$ to $2 \theta=50^{\circ}$ at a rate of $1 \% \mathrm{~min}$ [44].

374 Gel Permeation Chromatography (GPC) analysis. The molecular weight of PE

375 films treated by medium or fungus was determined by GPC on an Agilent PL-

376 GPC220 (Agilent Technologies, USA) equipped with Agilent PLgel Olexis $300 \times 7.5$

$377 \mathrm{~mm}$ columns and operating at $150{ }^{\circ} \mathrm{C}$ [45]. Trichlorobenzene was used as a mobile

378 phase $(1 \mathrm{~mL} / \mathrm{min})$ after calibration with polystyrene standards of known molecular

379 mass. A sample concentration of $1 \mathrm{mg} / \mathrm{mL}$ was used [43, 46].

380 Gas chromatography-mass spectrometry (GC-MS) analysis. The products of PE

381 biodegradation were detected by GC-MS. Briefly, after 60-day or 120-day incubation

382 of strain FB1 in the minimum medium supplemented with or without PE,

383 corresponding cell suspension was centrifuged $\left(12,000 \mathrm{~g}, 30 \mathrm{~min}, 4{ }^{\circ} \mathrm{C}\right)$ to collect the

384 supernatant. The supernatant was freeze-dried and re-dissolved in $1 \mathrm{~mL}$

385 dichloromethane, then $2 \mu \mathrm{L}$ filtered supernatant was used for GC-MS analysis 
386 performed on TRACE_1300GC-ISQ_LT GC-MS system (Shismadzu, Japan)

387 equipped with a TG-5ms (30 m long, $0.25 \mathrm{~mm}$ internal diameter and $0.25 \mu \mathrm{m}$ 388 thickness) chromatographic column [47]. The injection-port was set at $300{ }^{\circ} \mathrm{C}$. During 389 operation the column temperature was held for 4 min at $50{ }^{\circ} \mathrm{C}$, then raised to $300{ }^{\circ} \mathrm{C}$ 390 at $20^{\circ} \mathrm{C}$ rise per min, and finally, held for $15 \mathrm{~min}$ at $300{ }^{\circ} \mathrm{C}$. The flow rate was 0.800 $391 \mathrm{~mL} / \mathrm{min}$. Helium was used as a carrier gas. Ions/fragments were monitored in 392 scanning mode through 30-450 Amu.

393 Genomic and transcriptomic analyses. To sequence the genome of strain FB1, the 394 fungus was cultured in the PDA medium for 5 days, then the cells were collected and 395 total DNAs were extracted with a DNeasy Blood and Tissue Kit (Qiagen, Germany) 396 according to the instructions. Genomic sequencing was performed by Novogene 397 (Tianjin, China) [48]. Sequencing libraries were generated using NEBNext ${ }^{\circledR}$ Ultra $^{\mathrm{TM}}$ 398 DNA Library Prep Kit for Illumina (NEB, USA) following manufacturer's 399 recommendations and index codes were added to attribute sequences to each sample 400 [49]. Five databases were used to predict gene functions, including GO [50], KEGG 401 [51], COG [52], NR [53] and Swiss-Prot [54].

402 For the transcriptomic analysis, strain FB1 was cultured in the minimum medium 403 supplemented with or without PE films (type ET311350) for 45 d. Thereafter, the 404 fungal cells were collected for further transcriptomic analyses performed by 405 Novogene (Tianjin, China). Briefly, total RNAs from each sample were extracted and 406 RNA degradation and contamination were monitored on 1\% agarose gels. RNA purity 407 was checked using the NanoPhotometer ${ }^{\circledR}$ spectrophotometer (Implen, USA). RNA 408 concentration was measured using Qubit ${ }^{\circledR}$ RNA Assay Kit in Qubit® 2.0 Flurometer 409 (Life Technologies, USA). RNA integrity was assessed using the RNA Nano 6,000 410 Assay Kit of the Bioanalyzer 2100 system (Agilent Technologies, USA). A total 411 amount of $1 \mu \mathrm{g}$ RNA per sample was used as input material for the RNA sample 412 preparations. Sequencing libraries were generated using NEBNext® Ultra ${ }^{\mathrm{TM}}$ RNA 413 Library Prep Kit for Illumina ${ }^{\circledR}$ (NEB, USA) following manufacturer's 
414 recommendations and index codes were added to attribute sequences to each sample.

415 The clustering of the index-coded samples was performed on a cBot Cluster

416 Generation. After cluster generation, the library preparations were sequenced on an

417 Illumina Hiseq platform and $125 \mathrm{bp} / 150 \mathrm{bp}$ paired-end reads were generated. Raw

418 data (raw reads) of fastq format were firstly processed through in-house perl scripts

419 [55]. Reference genome and gene model annotation files were downloaded from

420 genome website directly. Index of the reference genome was built using Hisat2 v2.0.4

421 and paired end clean reads were aligned to the reference genome using Hisat2 v2.0.4.

422 HTSeq v0.9.1 was used to count the reads numbers mapped to each gene. And then

423 FPKM of each gene was calculated based on the length of the gene and reads count

424 mapped to this gene. Differential expression analysis was performed by using the

425 DESeq R package (1.18.0)[56]. Gene Ontology (GO) enrichment analysis of

426 differentially expressed genes was implemented by the GOseq R package, in which

427 gene length bias was corrected. KOBAS software was used to test the statistical

428 enrichment of differential expression genes in KEGG pathways[57, 58]. PPI analysis

429 of differentially expressed genes was based on the STRING database, which predicted

430 Protein-Protein Interactions. The Cufflinks v2.1.1 Reference Annotation Based

431 Transcript (RABT) assembly method was used to construct and identify both known

432 and novel transcripts from TopHat alignment results. Picard-tools v1.96 and samtools

$433 \mathrm{v} 0.1 .18$ were used to sort, mark duplicated reads and reorder the bam alignment

434 results of each sample. GATK2 (v3.2) software was used to perform SNP calling.

435 Expression, purification and functional assay of potential PE-degrading enzymes.

436 To verify the degradation effects of glutathione peroxidase (evm.model.contig_3.359)

437 and laccase (evm.model.contig_8.535) that identified in Alternaria sp. FB1, the genes

438 encoding these two proteins were respectively cloned and overexpressed in the E. coli

439 cells. First, the intact gene encoding glutathione peroxidase or laccase was amplified

440 from the cDNA template of strain FB1 using the KOD One TM PCR Master Mix

441 (TOYOBO, Japan) with corresponding primers (Supplementary Table S3). The PCR 
442 product was purified by using a DNA Gel Extraction Kit (TsingKe, China), and then

443 was cloned in the plasmid pMD19-T simple (TAKARA, Japan). The DNA fragment

444 was digested with BamHI/XhoI (Thermo Fisher Scientific, USA), respectively, and

445 ligated into corresponding sites of the expression vector pET28a(+) (Merck,

446 Germany). The recombinant plasmids were transformed into competent cells of $E$.

447 coli BL21(DE3) (TsingKe, China), and transformants were incubated in the Luria-

448 Bertani broth $(10 \mathrm{~g} \mathrm{NaCl}, 10 \mathrm{~g}$ tryptone and $5 \mathrm{~g}$ yeast extract per liter of Milli-Q

449 water) supplemented with $50 \mu \mathrm{g} / \mathrm{mL}$ kanamycin at $37{ }^{\circ} \mathrm{C}$. Protein expression was

450 induced at an OD600 around 0.6 with $0.1 \mathrm{mM}$ isopropyl-1-thio- $\beta$-D-

451 galactopyranoside (ITPG), and the cells were cultured for further $20 \mathrm{~h}$ at $16{ }^{\circ} \mathrm{C}$.

452 Recombinant proteins were purified with a HisTrapTM HP (GE Healthcare, Sweden)

453 by an AKTA pure system (GE Healthcare, Sweden), and dialyzed against filtered and

454 sterilized seawater for $4 \mathrm{~h}$. The purified proteins were checked by SDS-PAGE, and

455 visualized with Coomassie Bright Blue R250. The degradation effects were detected

456 in a solution containing respective proteins at a final concentration of $0.1 \mathrm{mg} / \mathrm{mL}$ with

457 PE films (type ET311350, $0.25 \mathrm{~mm}$ in thickness) at $30{ }^{\circ} \mathrm{C}$ for $48 \mathrm{~h}$. The surface

458 morphology and molecular weight of the PE films were respectively checked by SEM

459 and GPC as described above.

460 Data availability. The complete genome sequence of Alternaria sp. FB1 has been

461 deposited at GenBank under the accession number PRJNA672824. Raw sequencing

462 reads for transcriptomic analysis have been deposited at NCBI under accession

463 numbers SRR15043810 and SRR15043809. Mass spectrometry analyses of

464 components in 60-day fungal treatment sample at different retention times were

465 shown in Supplementary Figures S6-S12. Mass spectrometry analyses of components

466 in 120-day fungal treatment sample at different retention times were shown in

467 Supplementary Figures S13-S25. 


\section{Acknowledgements}

470 This work is funded by the Major Research Plan of the National Natural Science 471 Foundation (Grant No. 92051107), Key Deployment Projects of Center of Ocean 472 Mega-Science of the Chinese Academy of Sciences (Grant No. COMS2020Q04), 473 National Key R and D Program of China (Grant No. 2018YFC0310800), Strategic 474 Priority Research Program of the Chinese Academy of Sciences (Grant No. 475 XDA22050301), and Taishan Young Scholar Program of Shandong Province 476 (tsqn20161051) for Chaomin Sun.

\section{Author Contributions}

478 RG and CS conceived and designed the study. RG performed most of experiments. 479 RL helped to purify proteins. RG and CS analyzed the data. RG wrote the manuscript. 480 CS revised the manuscript. All authors read and approved the final manuscript.

\section{Conflict of interest}

482 The authors have no conflict of interest.

483

484

485

486

487

488

489

490

491

492

493

494

495

496

497

498

499

500

\section{References}

1. Kurniawan, S.B., Abdullah, S.R.S., Imron, M.F., and Ismail, N.I. (2021). Current state of marine plastic pollution and its technology for more eminent evidence: A review. J Clean Prod 278.

2. Millican, J.M., and Agarwal, S. (2021). Plastic pollution: a material problem? Macromolecules 54, 4455-4469.

3. Peplow, M. (2019). Ocean survey tracks rising plastic pollution. Chem Eng News 97, 1010.

4. Rhodes, C.J. (2018). Plastic pollution and potential solutions. Sci Progress-Uk 101, 207260.

5. Chen, Y., Awasthi, A.K., Wei, F., Tan, Q.Y., and Li, J.H. (2021). Single-use plastics: Production, usage, disposal, and adverse impacts. Sci Total Environ 752.

6. Geyer, R., Jambeck, J.R., and Law, K.L. (2017). Production, use, and fate of all plastics ever made. Sci Adv 3.

7. Harshvardhan, K., and Jha, B. (2013). Biodegradation of low-density polyethylene by marine bacteria from pelagic waters, Arabian Sea, India. Mar Pollut Bull 77, 100-106.

8. Hakkarainen, M., and Albertsson, A.-C. (2004). Environmental Degradation of Polyethylene. In Long Term Properties of Polyolefins. pp. 177-200. 
501

502

503

504

505

506

507

508

509

510

511

512

513

514

515

516

517

518

519

520

521

522

523

524

525

526

527

528

529

530

531

532

533

534

535

536

537

538

539

540

541

9. Vimala, P.P., and Mathew, L. (2016). Biodegradation of Polyethylene Using Bacillus Subtilis. Procedia Tech 24, 232-239.

10. Brandon, A.M., Gao, S.H., Tian, R., Ning, D., Yang, S.S., Zhou, J., Wu, W.M., and Criddle, C.S. (2018). Biodegradation of Polyethylene and Plastic Mixtures in Mealworms (Larvae of Tenebrio molitor) and Effects on the Gut Microbiome. Environ Sci Technol $52,6526-6533$.

11. Zahra, S., Abbas, S.S., Mahsa, M.T., and Mohsen, N. (2010). Biodegradation of lowdensity polyethylene (LDPE) by isolated fungi in solid waste medium. Waste Manag 30, 396-401.

12. Veethahavya, K.S., Rajath, B.S., Noobia, S., and Kumar, B.M. (2016). Biodegradation of Low Density Polyethylene in Aqueous Media. Procedia Environ Sci 35, 709-713.

13. Bardaji, D.K.R., Moretto, J.A.S., Furlan, J.P.R., and Stehling, E.G. (2020). A minireview: current advances in polyethylene biodegradation. World J Microb Biot 36.

14. Liu, J.W., He, J., Xue, R., Xu, B., Qian, X.J., Xin, F.X., Blank, L.M., Zhou, J., Wei, R., Dong, W.L., et al. (2021). Biodegradation and up-cycling of polyurethanes: Progress, challenges, and prospects. Biotechnol Adv 48.

15. Santacruz-Juarez, E., Buendia-Corona, R.E., Ramirez, R.E., and Sanchez, C. (2021). Fungal enzymes for the degradation of polyethylene: Molecular docking simulation and biodegradation pathway proposal. J Hazard Mater 411.

16. Sanchez, C. (2020). Fungal potential for the degradation of petroleum-based polymers: An overview of macro- and microplastics biodegradation. Biotechnol Adv 40.

17. Shin, J., Kim, J.E., Lee, Y.W., and Son, H. (2018). Fungal cytochrome P450s and the P450 complement (CYPome) of Fusarium graminearum. Toxins 10.

18. Sangale, M.K., Shahnawar, M., and Ade, A.B. (2019). Potential of fungi isolated from the dumping sites mangrove rhizosphere soil to degrade polythene. Sci Rep-Uk 9.

19. Lucas, N., Bienaime, C., Belloy, C., Queneudec, M., Silvestre, F., and Nava-Saucedo, J.E. (2008). Polymer biodegradation: Mechanisms and estimation techniques. Chemosphere $73,429-442$.

20. Gilan, I., Hadar, Y., and Sivan, A. (2004). Colonization, biofilm formation and biodegradation of polyethylene by a strain of Rhodococcus ruber. Appl Microbiol Biot 65, 97-104.

21. Zhang, J.Q., Gao, D.L., Li, Q.H., Zhao, Y.X., Li, L., Lin, H.F., Bi, Q.R., and Zhao, Y.C. (2020). Biodegradation of polyethylene microplastic particles by the fungus Aspergillus flavus from the guts of wax moth Galleria mellonella. Sci Total Environ 704.

22. Tribedi, P., and Sil, A.K. (2013). Low-density polyethylene degradation by Pseudomonas sp AKS2 biofilm. Environ Sci Pollut R 20, 4146-4153.

23. Karich, A., Ullrich, R., Scheibner, K., and Hofrichter, M. (2017). Fungal Unspecific Peroxygenases Oxidize the Majority of Organic EPA Priority Pollutants. Front Microbiol 8.

24. Schwartz, M., Perrot, T., Aubert, E., Dumarcay, S., Favier, F., Gerardin, P., MorelRouhier, M., Mulliert, G., Saiag, F., Didierjean, C., et al. (2018). Molecular recognition of 
542

543

544

545

546

547

548

549

550

551

552

553

554

555

556

557

558

559

560

561

562

563

564

565

566

567

568

569

570

571

572

573

574

575

576

577

578

579

580

581

582

583

wood polyphenols by phase II detoxification enzymes of the white rot Trametes versicolor. Sci Rep-Uk 8.

25. Restrepo-Florez, J.M., Bassi, A., and Thompson, M.R. (2014). Microbial degradation and deterioration of polyethylene - A review. Int Biodeter Biodegr 88, 83-90.

26. Wei, R., and Zimmermann, W. (2017). Microbial enzymes for the recycling of recalcitrant petroleum-based plastics: how far are we? Microb Biotechnol 10, 1308-1322.

27. Jambeck, J.R., Geyer, R., Wilcox, C., Siegler, T.R., Perryman, M., Andrady, A., Narayan, R., and Law, K.L. (2015). Plastic waste inputs from land into the ocean. Science 347, 768-771.

28. Rosato, A., Barone, M., Negroni, A., Brigidi, P., Fava, F., Xu, P., Candela, M., and Zanaroli, G. (2020). Microbial colonization of different microplastic types and biotransformation of sorbed PCBs by a marine anaerobic bacterial community. Sci Total Environ 705.

29. Gao, R.R., and Sun, C.M. (2021). A marine bacterial community capable of degrading poly(ethylene terephthalate) and polyethylene. J Hazard Mater 416.

30. Bombelli, P., Howe, C.J., and Bertocchini, F. (2017). Polyethylene bio-degradation by caterpillars of the wax moth Galleria mellonella. Curr Biol 27, R292-R293.

31. Ameen, F., Moslem, M., Hadi, S., and Al-Sabri, A.E. (2015). Biodegradation of 1

ow density polyethylene (LDPE) by mangrove fungi from the Red Sea coast. Prog Rubber Plast Re 31, 125-143.

32. Chen, A.Q., Mao, X., Sun, Q.H., Wei, Z.X., Li, J., You, Y.L., Zhao, J.Q., Jiang, G.B., Wu, Y.N., Wang, L.P., et al. (2021). Alternaria mycotoxins: an overview of toxicity, metabolism, and analysis in food. J Agr Food Chem 69, 7817-7830.

33. Dixit, O., and Mollekopf, N. (2014). Designing absorption processes with aqueous Diglycolamine. Chem Eng Technol 37, 1583-1592.

34. Pal, P., Shittu, I., Othman, I., Sengupta, A., Voleti, L.D., and Banat, F. (2020). Removal of the total organic acid anions from an industrial lean diglycolamine solvent using a calcium alginate carbon adsorbent, and molecular modeling studies. J Nat Gas Sci Eng 82.

35. Ali, S.S., Elsamahy, T., Al-Tohamy, R., Zhu, D.C., Mahmoud, Y.A.G., Koutra, E., Metwally, M.A., Kornaros, M., and Sun, J.Z. (2021). Plastic wastes biodegradation: Mechanisms, challenges and future prospects. Sci Total Environ 780.

36. Santo, M., Weitsman, R., and Sivan, A. (2013). The role of the copper-binding enzyme laccase - in the biodegradation of polyethylene by the actinomycete Rhodococcus ruber. Int Biodeter Biodegr 84, 204-210.

37. Spina, F., Tummino, M.L., Poli, A., Prigione, V., Ilieva, V., Cocconcelli, P., Puglisi, E., Bracco, P., Zanetti, M., and Varese, G.C. (2021). Low density polyethylene degradation by filamentous fungi. Environ Pollut 274.

38. Jeon, H.J., and Kim, M.N. (2015). Functional analysis of alkane hydroxylase system derived from Pseudomonas aeruginosa E7 for low molecular weight polyethylene biodegradation. Int Biodeter Biodegr 103, 141-146.

39. van Beilen, J.B., Li, Z., Duetz, W.A., Smits, T.H.M., and Witholt, B. (2003). Diversity of alkane hydroxylase systems in the environment. Oil Gas Sci Technol 58, 427-440. 
584

585

586

587

588

589

590

591

592

593

594

595

596

597

598

599

600

601

602

603

604

605

606

607

608

609

610

611

612

613

614

615

616

617

618

619

620

621

622

623

624

625

40. Christensen, B.E., Trønnes, H.N., Vollan, K., Smidsrød, O., and Bakke, R. (1990). Biofilm removal by low concentrations of hydrogen peroxide. Biofouling 2, 165-175.

41. Sammon, C., Yarwood, J., and Everall, N. (2000). A FTIR-ATR study of liquid diffusion processes in PET films: comparison of water with simple alcohols. Polymer 41, 25212534.

42. Kardas, I., Lipp-Symonowicz, B., and Sztajnowski, S. (2011). The influence of enzymatic treatment on the surface modification of PET fibers. J Appl Polym Sci 119, 3117-3126.

43. Bonhomme, S., Cuer, A., Delort, A.M., Lemaire, J., Sancelme, M., and Scott, G. (2003). Environmental biodegradation of polyethylene. Polym Degrad Stabil 81, 441-452.

44. Xiao, Z.C., and Akpalu, Y.A. (2007). New insights into the characteristics of early stage crystallization of a polyethylene. Polymer 48, 5388-5397.

45. Huang, H.H., Guo, M.F., Wei, D., Suarez, I., Coto, B., Lopez, E., Ortin, A., and Yau, W.W. (2015). Direct comparison of IR and DRI detector for HT-GPC of polyolefins. Macromol Symp 356, 95-109.

46. Tung, L.H., and Buckser, S. (1959). The effect of molecular weight on the crystallinity of polyethylene. J Phys Chem-Us 62, 1530-1534.

47. Ren, L., Men, L.N., Zhang, Z.W., Guan, F.F., Tian, J., Wang, B., Wang, J.H., Zhang, Y.H., and Zhang, W. (2019). Biodegradation of polyethylene by Enterobacter sp. D1 from the guts of wax moth Galleria mellonella. Int J Env Res Pub He 16.

48. Zhang, J., Liu, R., Xi, S.C., Cai, R.N., Zhang, X., and Sun, C.M. (2020). A novel bacterial thiosulfate oxidation pathway provides a new clue about the formation of zero-valent sulfur in deep sea. ISME J. 14, 2261-2274.

49. Bankevich, A., Nurk, S., Antipov, D., Gurevich, A.A., Dvorkin, M., Kulikov, A.S., Lesin, V.M., Nikolenko, S.I., Pham, S., Prjibelski, A.D., et al. (2012). SPAdes: a new genome assembly algorithm and its applications to single-cell sequencing. J Comput Biol 19, 455477.

50. Ashburner, M., Ball, C.A., Blake, J.A., Botstein, D., Butler, H., Cherry, J.M., Davis, A.P., Dolinski, K., Dwight, S.S., Eppig, J.T., et al. (2000). Gene Ontology: tool for the unification of biology. Nat Genet 25, 25-29.

51. Kanehisa, M., Goto, S., Kawashima, S., Okuno, Y., and Hattori, M. (2004). The KEGG resource for deciphering the genome. Nucleic Acids Res 32, D277-D280.

52. Galperin, M.Y., Makarova, K.S., Wolf, Y.I., and Koonin, E.V. (2015). Expanded microbial genome coverage and improved protein family annotation in the COG database. Nucleic Acids Res 43, D261-D269.

53. Saier, M.H., Reddy, V.S., Tamang, D.G., and Vastermark, A. (2014). The transporter classification database. Nucleic Acids Res 42, D251-D258.

54. Bairoch, A., and Apweiler, R. (2000). The SWISS-PROT protein sequence database and its supplement TrEMBL in 2000. Nucleic Acids Res 28, 45-48.

55. Wang, Z., Gerstein, M., and Snyder, M. (2009). RNA-Seq: a revolutionary tool for transcriptomics. Nat Rev Genet 10, 57-63.

56. Anders, S., and Huber, W. (2010). Differential expression analysis for sequence count data. Genome Biol 11. 
626

627

628

629

630

631

632

633

634

635

636

637

638

639

640

641

642

643

644

645

646

647

648

649

650

651

652

653

654

655

656

657

658

659

660

661

662

663

664

665

666

667
57. Kanehisa, M., Araki, M., Goto, S., Hattori, M., Hirakawa, M., Itoh, M., Katayama, T., Kawashima, S., Okuda, S., Tokimatsu, T., et al. (2008). KEGG for linking genomes to life and the environment. Nucleic Acids Res 36, D480-D484.

58. Mao, X.Z., Cai, T., Olyarchuk, J.G., and Wei, L.P. (2005). Automated genome annotation and pathway identification using the KEGG Orthology (KO) as a controlled vocabulary. Bioinformatics 21, 3787-3793. 
668 Figure Legends

669 Fig. 1 SEM observation of colonization and degradation effects of Alternairia sp.

670 FB1 on the PE film. a, SEM observation of the PE film treated by the medium only

671 for 7 days. b-d, SEM observation of the colonization of strain FB1 on the PE film

672 after 7 days treatment. e, SEM observation of the PE film treated by the medium for

673120 days. f-h, SEM observation of the degradation effects of strain FB1 on the PE

674 film after 120 days treatment.

675 Fig. 2 Significant morphological change of the PE film treated by Alternairia sp.

676 FB1. a, Morphology of the PE film treated by the medium for 120 days. b,

677 Morphology of the PE film treated by strain FB1 for 3 days. c, Morphology of the PE

678 film treated by strain FB1 for 120 days. For panels a-c, the PE film was soaked in the

679 medium without or with strain FB1. d, Morphology of the PE film treated by strain

680 FB1 for 120 days. e, An amplifying observation of panel d. For panel d, the culture of

681 strain FB1 was incubated in some area of the PE film. During the incubation course,

682 proper amount of fresh medium was supplemented to maintain the growth of strain 683 FB1.

684 Fig. 3 Verification of degradation effects of the PE film by Alternairia sp. FB1. a, 685 FTIR analysis of the PE film treated by the medium without or with strain FB1 for 686 two and four weeks. b, XRD analysis of the PE film treated by the medium without or 687 with strain FB1 for four weeks. c, d GPC analysis of the PE film treated by the 688 medium without (c) or with (d) strain FB1 for120 days.

689 Fig. 4 GC-MS analysis of products released from the PE film treated by 690 Alternairia sp. FB1. a, The carbon number and respective proportion of degradation 691 products released from the PE film treated by strain FB1 for 60 days. b, The carbon 692 number and respective proportion of degradation products released from the PE film 693 treated by strain FB1 for 120 days. c, The chemical formula, component, proportion 
694 and chemical structure of major products released from the PE film treated by strain

695 FB1 for 60 days and 120 days.

696 Fig. 5 Transcriptomic analyses of PE degradation processes directed by

697 Alternairia sp. FB1. a, A heat map showing the significantly up-regulated genes

698 encoding enzymes with potential PE-degradation activities in strain FB1 that

699 incubated with the PE film for 45 days. The number after corresponding enzymes'

700 name indicates the amount of genes whose expressions were significantly up-

701 regulated (cutoff $>2$ folds). b, SEM observation of the PE film treated by the

702 sterilized seawater for 48 h. c, SEM observation of the PE film treated by $0.1 \mathrm{mg} / \mathrm{mL}$

703 glutathione peroxidase (evm.model.contig_3.359) at $30{ }^{\circ} \mathrm{C}$ for $48 \mathrm{~h}$. d, SEM

704 observation of the PE film treated by $0.1 \mathrm{mg} / \mathrm{mL}$ laccase (evm.model.contig_8.535) at

$70530{ }^{\circ} \mathrm{C}$ for $48 \mathrm{~h}$. e, SEM observation of the PE film treated by both $0.1 \mathrm{mg} / \mathrm{mL}$

706 glutathione peroxidase (evm.model.contig_3.359) and $0.1 \mathrm{mg} / \mathrm{mL}$ laccase

707 (evm.model.contig_8.535) at $30{ }^{\circ} \mathrm{C}$ for $48 \mathrm{~h}$. f, GPC analysis of the PE film treated by

708 the sterilized seawater for $48 \mathrm{~h}$. g, GPC analysis of the PE film treated by $0.1 \mathrm{mg} / \mathrm{mL}$

709 glutathione peroxidase (evm.model.contig_3.359) at $30{ }^{\circ} \mathrm{C}$ for $48 \mathrm{~h}$. h, GPC analysis

710 of the PE film treated by $0.1 \mathrm{mg} / \mathrm{mL}$ laccase (evm.model.contig_8.535) at $30{ }^{\circ} \mathrm{C}$ for

$71148 \mathrm{~h}$. i, GPC analysis of the PE film treated by both $0.1 \mathrm{mg} / \mathrm{mL}$ glutathione

712 peroxidase (evm.model.contig_3.359) and $0.1 \quad \mathrm{mg} / \mathrm{mL}$ laccase

713 (evm.model.contig_8.535) at $30{ }^{\circ} \mathrm{C}$ for $48 \mathrm{~h}$.

714 Fig. 6 A proposed PE biodegradation model by the fungus. There are four stages

715 in the PE biodegradation process: colonization/erosion, depolymerization,

716 assimilation and mineralization. In this process, the PE polymer was degraded into

717 small fragments step by step, then finally was converted to energy, carbon source as

718 well as the $\mathrm{CO}_{2}$ and $\mathrm{H}_{2} \mathrm{O}$. The detailed description of this model was shown in the

719 results part.

720 







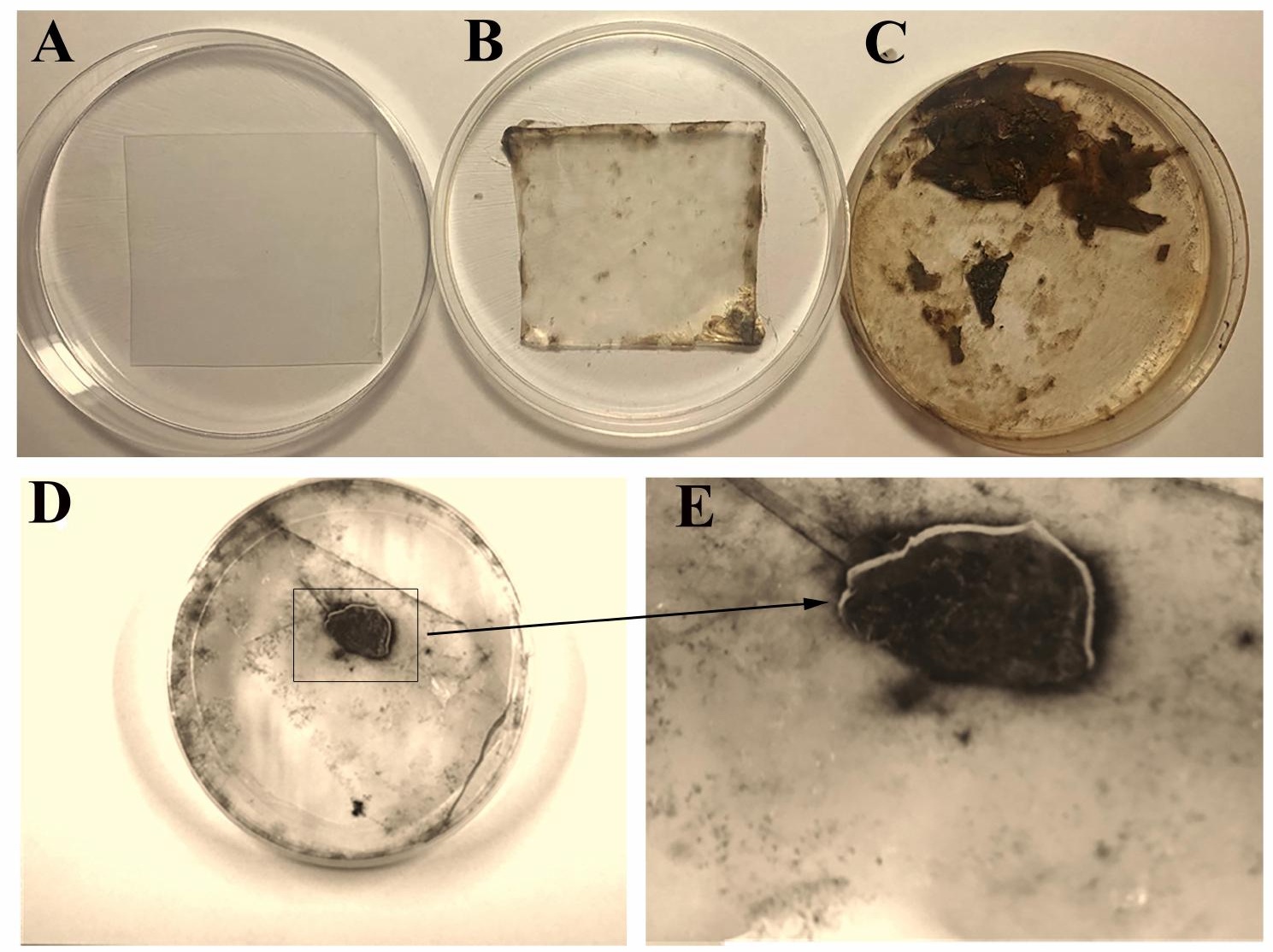



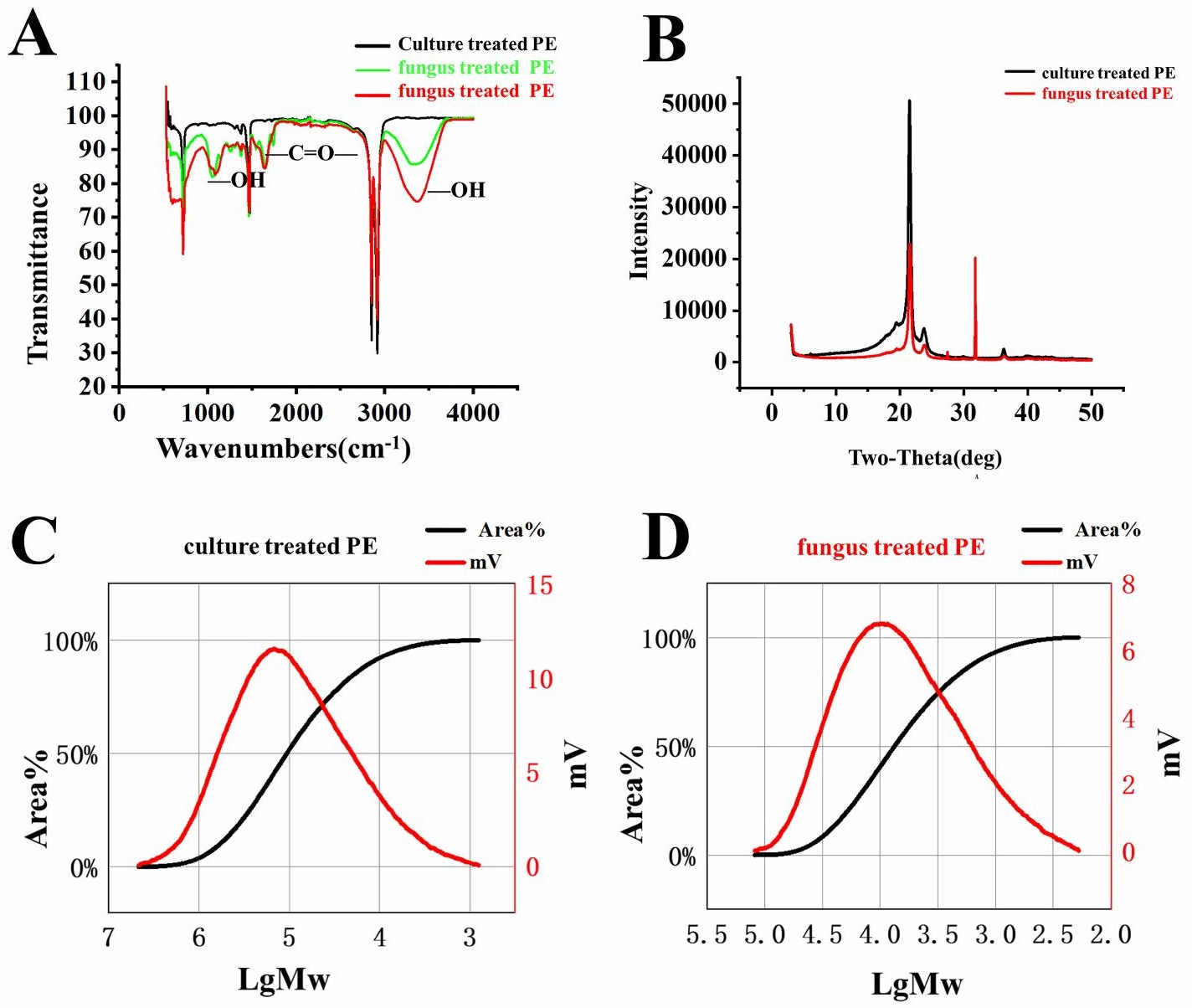

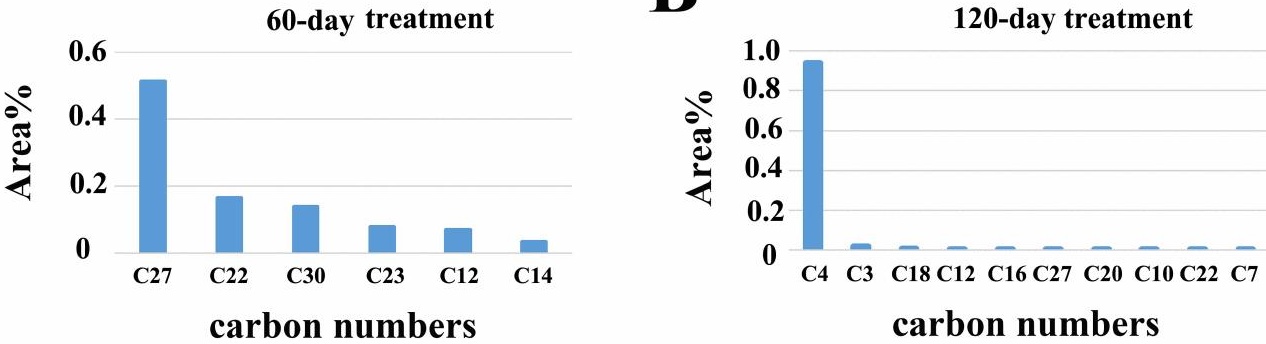

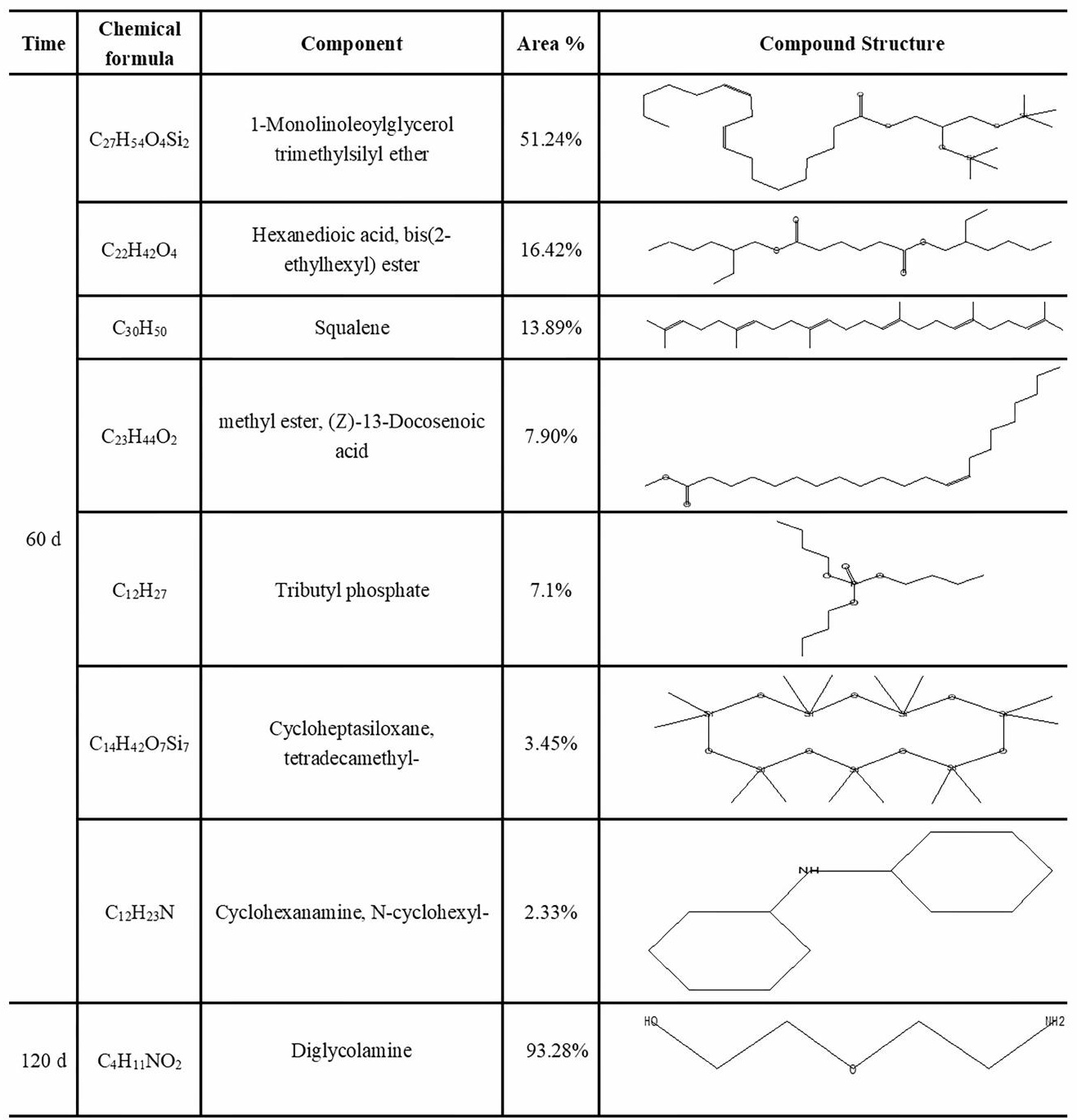



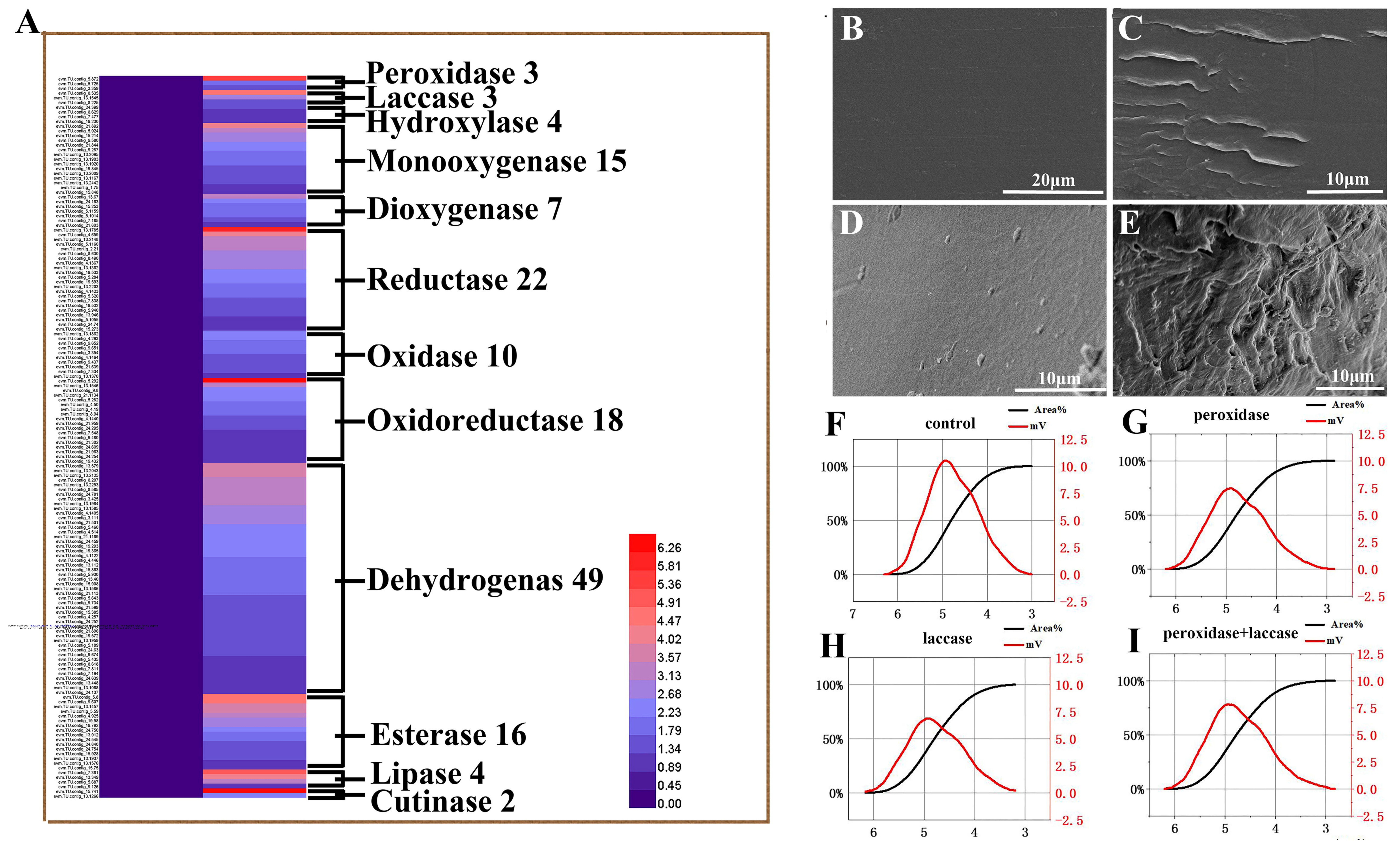


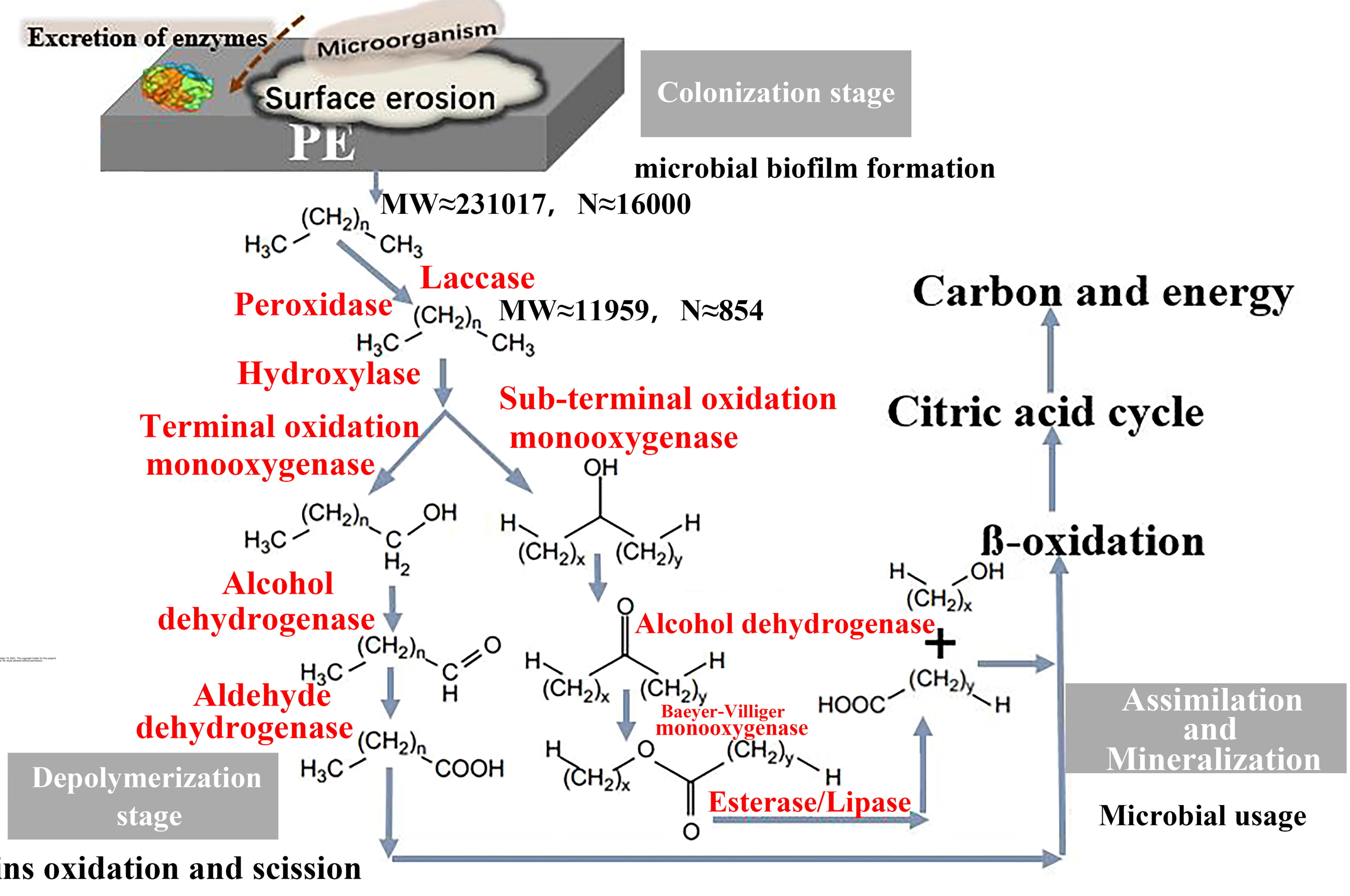

